Dept. of Math. University of Oslo

\title{
An Adaptive Method for Evaluating Multidimensional Contingent Claims. Part II
}

Lars O. Dahl

28th February 2002

Department of Mathematics, Norwegian University of Science and Technology, N-7491 Trondheim, Norway and Storebrand Investments, PO Box 1380, N-0114 Oslo, Norway (correspondence address) Email: lars.oswald.dahl@storebrand.com

Url: http://www.math.ntnu.no/ larsosw

\begin{abstract}
This is part two of a work on adaptive integration methods aimed at multidimensional option pricing problems in finance. It presents simulation results of an adaptive method developed in the companion article [3] for the evaluation of multidimensional integrals over the unit cube. The article focuses on a rather general test problem constructed to give insights in the success of the adaptive method for option pricing problems. We establish a connection between the decline rate of the ordered eigenvalues of the pricing problem and the efficiency of the adaptive method relative to the non-adaptive. This gives criteria for when the adaptive method can be expected to outperform the non-adaptive for other pricing problems. In addition to evaluating the method for different problem parameters, we present simulation results after adding various techniques to enhance the adaptive method itself. This includes using variance reduction techniques for each sub-problem resulting from the partitioning of the integration domain. All simulations are done with both pseudo-random numbers and quasi-random numbers (low discrepancy sequences), resulting in Monte Carlo (MC) and quasi-Monte Carlo (QMC) estimators and the ability to compare them in the given setting.

The results show that the adaptive method can give performance gains in the order of magnitudes for many configurations, but it should not be used incautious, since this ability depends heavily on the problem at hand.
\end{abstract}

\section{Introduction}

The fair value of contingent claims can be expressed as an expected value, which in turn can be written as an integral. In many situations these integrals are multidimensional. The value of the integrand can vary significantly in the domain of integration, and in some cases there can be only small parts of the domain in which the integrand is non-zero. Since MC and QMC methods distribute the evaluation points as evenly as possible, they will waste calculations on regions which are not important. An adaptive method tries to allocate the resources to the important parts of the domain.

In option pricing problems where the option is written on several underlying assets and/or the option price is path-dependent in time, the price is found by calculating a multidimensional integral. QMC methods are often deployed for these problems, and additional methods for reducing the variance of the estimator for the price of the option can be found. The adaptive technique incorporates some common variance reduction principles implicitly, but the modifications of the integrand are not exactly the same. In particular, the adaptive method is 
closely related to importance sampling and stratified sampling. References on these methods are [7], [6], [8], [9], [17], [20], [22]. The purpose of these methods, as the adaptive, is to use the samples from the QMC generator in a more effective way than distributing them evenly in the domain. Some knowledge of the integrand must be present, or collected, for the success of these methods. Importance sampling can be dangerous in that one is not guaranteed lower variance. The variance can actually blow up for special cases. The advantage of the adaptive method is that information is collected as the calculation is done, and function evaluations of the integrand is used to guide the distribution of the points in the domain.

In this article we design a parameterized test problem which has similarities with option pricing problems. We do a number of numerical simulations on this problem for various parameters of the problem, and various adjustments of the adaptive method. The purpose is to find good criteria for when the adaptive method can be expected to outperform a conventional method for different problems. In particular we experiment with local control variates in each sub-domain from the adaption process. Furthermore, we investigate the effect of using the Halton Leaped low discrepancy sequence compared to the Mersenne Twister pseudo random sequence as an engine for the resulting QMC respectively $\mathrm{MC}$ methods, and look at the impact these methods have on the adaptive method compared to the non-adaptive method. We test the methods on the problem after applying importance sampling to investigate if the performance of the methods are affected by this. The use of variance reduction techniques in local domains and the use of MC and QMC for the evaluation of the sub-problems resulting from the adaption process is to our knowledge not investigated before. The numerical tests show that the adaptive method has good performance compared to non-adaptive methods for many problems. In particular we get good results for out-of-themoney options and general functions having different behavior in different parts of the domain. Furthermore we are able to identify easy implementable criteria for when the adaptive method is preferable over the non-adaptive method.

In sec. 2 we describe a finance problem suited as an example to test the algorithms. In sec. 3 we generalize this problem by parameterizing it, and describe how to find the introduced parameter for general option pricing problems with the same structure. We show that the introduced parameter can be used to characterize real world problems in sec. 4 , and we introduce two efficiency measures for the methods in sec. 5 . In sec. 6 and sec. 7 we give numerical results, and in sec. 8 we elaborate further on the findings by looking at two functions with less resemblance to finance.

\section{Pricing of exotic options}

The integrand $f(\cdot)$ in our problem is a function in $C^{0}\left([0,1]^{s}\right)$ and is, in its initial form, monotone in each variable. The different variance reduction techniques 
described below can alter the monotonic behavior, but the continuity is maintained. Motivated from applications of finance, we may assume in this paper that the function has only one hyper-plane in which it is not differentiable, and that it in the rest of the domain is in $C^{\infty}\left([0,1]^{s}\right)$. Note that the method is applicable for more general integrands, but this would involve a more complicated and time consuming routine to investigate the integration domain to insure a proper subdivision. The method used in this paper is tailored for finance problems, and enables us to get proper subdivisions by using relatively few resources.

We have chosen to investigate the pricing problem of an Asian option on multiple underlying assets as an example (called an Asian basket option). The adaptive method is also suited for other option contracts which can be formulated as an integration problem, and is especially successful for problems where it is possible to identify a subset of dimensions/variables for which the integrand is most dependent, and problems where the integrand is zero or constant in parts of the domain. We give a brief description of the Asian basket option pricing problem below.

We operate in the context of a complete, standard financial market with constant risk-free rate $r$ and constant volatility, see e.g. [10] for a definition of such a market. A stock in this market is modeled by

$$
S_{n}(t)=S_{n}(0) \exp \left(P_{n}+\sum_{d=1}^{N} \sigma_{n d} W_{0}^{d}(t)\right), n=1, \ldots, N,
$$

where $\sigma$ is the volatility matrix of the $N$ assets in the market and the stochastic process $W_{0}(t)$ is an $N$ dimensional Brownian motion under the risk free measure. The term $P_{n}$ is introduced for easier notation and is given by $\left(r-\frac{1}{2} \sum_{d=1}^{N} \sigma_{n d}^{2}\right) t$. Without going into details (which can be found in [10]), we state that the value at time $t$ of an Asian option is

$$
V(t)=e^{-r(T-t)} \mathrm{E}_{0}[\varphi(\Upsilon(\mathcal{T})) \mid \mathcal{F}(t)]
$$

where $\varphi(\cdot)$ is a Borel measurable function given by $\varphi(Y)=(Y-q)^{+}$, resulting in the European-style Asian option with strike $q$. Furthermore we are interested in $V(t)$ at $t=0$, i.e. at the time the option is bought. The argument $\mathcal{T}$ is a vector of discrete points in time $\mathcal{T}=\left(t_{0}, t_{1}, \ldots, t_{K}\right), t_{K}=T$, where the number of sampling points $K+1$ is specified. The length of the intervals $t_{k}-t_{k-1}$ need not be equal. For a discussion of an even more general setup, see e.g. [21]. The $\Upsilon$ function for the Asian basket option is given by

$$
\Upsilon(\mathcal{T})=\frac{1}{N(K+1)} \sum_{k=0}^{K} \sum_{n=1}^{N} S_{n}\left(t_{k}\right)
$$

The price of the Asian basket option thus becomes:

$$
V(0)=e^{-r T} \mathrm{E}_{0}[\varphi(\Upsilon(\mathcal{T}))]
$$


In order to replace the expected value in (4) with an integral over $[0,1]^{D}$, where $D=N(K+1)$, and to be able to solve the integral effectively with QMC methods and apply the adaptive approach, we must formulate $\varphi(\Upsilon(\mathcal{T}))$ in terms of independent stochastic processes. This can be done by constructing the $\sigma$ matrix from an SV-decomposition of the covariance matrix of returns for the assets over some period, and by performing an SV-decomposition of the covariance structure of the discretized Brownian motion to simulate the $W\left(t_{k}\right)$ process. See e.g. [16] on the Brownian motion or [4] for the full treatment with regards to the Asian basket option. We then obtain a formulation where we have identified the eigenvalues of $\sigma^{2}$, and are able to give priority to the parts of the problem corresponding to the biggest eigenvalues. The unveiling and ordering of the eigenvalues leads to a formulation where the integrand is most volatile along the first dimensions, with decreasing volatility for consecutive dimensions. Note that there are other approaches to achieve a formulation in terms of independent stochastic processes for path dependent options (see [4]), but the method described here is the only one for which we are able to identify the most important sources of noise for the full problem, and therefore the best suited model for the adaptive approach. To expose the independent stochastic processes we introduce the notation $S\left(t_{k}, \varepsilon_{k}\right)$. The price of the option at $t=0$ can then be written

$$
\begin{aligned}
V(0) & =e^{-r T} \int_{\mathbb{R}^{D}} \varphi\left(\frac{1}{D} \sum_{k=0}^{K} \sum_{n=1}^{N} S_{n}\left(t_{k}, x\right)\right) \psi(x) d x \\
& =e^{-r T} \int_{[0,1]^{D}} \varphi\left(\frac{1}{D} \sum_{k=0}^{K} \sum_{n=1}^{N} S_{n}\left(t_{k}, \varepsilon(y)\right)\right) d y,
\end{aligned}
$$

where $\psi: \mathbb{R}^{D} \rightarrow \mathbb{R}^{D}$ is the density of a $D$ - dimensional centered Gaussian random variable with covariance matrix equal to the identity, and $\varepsilon:[0,1]^{D} \rightarrow \mathbb{R}^{D}$ is a vector of inverse cumulative normal distribution functions with mean 0 and variance 1: $\varepsilon(y)=\left\langle\varepsilon_{1}\left(y_{1}\right), \ldots, \varepsilon_{D}\left(y_{D}\right)\right\rangle$. We do the evaluation of $\varepsilon(\cdot)$ by a rational approximation suggested in [12]. The final problem formulation in (6) is suitable for the adaptive algorithm. Note that the independent stochastic variables in the problem represents the coordinate axis of the integration problem, and the identification and ordering of the orthogonal noise components leads to a problem formulation where the integrand is "most active" in an area of the domain as small as possible. Furthermore the activity is localized to as few quadrants as possible, which also are neighbors. These characteristics are all favorable to the adaptive method. In the next section we will look at how the eigenvalues of the single asset Asian problem is altered by changing the time discretization, and use this to formulate a more general test problem suited for testing the performance of the adaptive method for general option pricing problems. 


\section{Parameterizing the Noise Structure of the Option Problem}

We know from previous work in e.g. [1], and from tests of our own algorithm, that the adaptive approach is not always competitive with conventional MC and QMC methods. The integrand need to have special characteristics as the dimension becomes high for this to be the case. The adaptive method should therefore not be used incautious. We want to formulate criteria for deciding when the adaptive method is competitive with conventional QMC for general option pricing and hedging problems. These problems are often on a similar form as the problem described in sec. 2, and the model of the underlying assets for a range of problems can generally be written as

$$
S_{n}=S_{n} \exp \left(P_{n}+C_{n} \varepsilon\right) ; 1 \leq n \leq N
$$

where $C_{n}$ is the $n^{\prime}$ th row of a positive semidefinite matrix $C$ and $\varepsilon$ is vector of independent normal distributed variables with length $N$. The matrix $C$ can be constructed by setting $C=\Sigma \Lambda^{1 / 2}$, where $\Lambda$ is the eigenvalue matrix of $C^{2}$, and $\Sigma$ is the matrix of eigenvectors. The matrix $C^{2}$ is a parameter depending on the market and the type of option to be priced. In the single asset Asian option problem $C^{2}$ is only depending on the form of the time discretization, and we can therefore use this problem to alter the contents of $C$, and to find a criterion based on the eigenvalues of $C^{2}$ to measure the relative efficiency of the adaptive approach.

We have formulated a functional behavior of the time discretization as

$$
f(t, a)=t^{a}, t \in[0,1] \text { and } a \in[0.001,1] .
$$

When the time discretization given by this function is applied to the pricing of the asian option, each value of $a$ give a different set of eigenvalues and eigenvectors for the matrix $C^{2}$. In fig. 1 we have plotted the square root of the normed eigenvalues in descending order for a typical configuration together with the function

$$
l(i)=i^{b}, i=1, \ldots, D, b \leq 0,
$$

where the parameter $b$ is found by a least squares fit of the function to the square root of the eigenvalues in descending order. As can be seen from the graphs, the fitted function is rather similar to the graphed square root of the eigenvalues. To extend the discussion to the range of $b>-1.37$ in (9) not captured by the adjustment of $a$ in (8) we have constructed an artificial parameterized problem class where we start out with the Asian option problem with $D$ equidistributed time discretization points, and the parameter $a$ equal to 1 . The resulting $C$ matrix of this problem is changed by exchanging the $\Lambda$ matrix with a new $\hat{\Lambda}$ matrix where the diagonal elements are $\lambda_{1} l(i), \lambda_{1}$ being the largest eigenvalue of the 
original $C^{2}$ matrix. By simulating on this artificial problem for $b \in[-1.4,0]$ we can extend the range in which we can evaluate the relative performance of the adaptive method. The reason for switching approach is that we are not able to produce eigenvalue dependence in the range $b<1.37$ for any $a$ in (8). As $a$ is increased above one, $b$ will increase rather than decrease further. In fig. 1 we have illustrated the dependence between $a$ and $b$.

Since option pricing problems often share the structure described above, and the behavior of the square root of the eigenvalues can be matched satisfactorily with the function $l(i)$, the conclusion of our numerical tests can be used to decide when to employ the adaptive method for new problems. The graphed results to illustrate the relative performance of the adaptive method will be done as a function of $b$ rather than $a$, because the parameter $b$ is the one that can be extracted from general option pricing problems.

\section{Comparison with actual pricing problems}

The parameterization in sec. 3 turned out to give a very close relationship between the rate of decline of the square root of the ordered eigenvalues and the function given in $l(i)=i^{b}$ for a fitted $b$. To argue that the survey we have performed can be put to use, we give the eigenvalues and the appurtenant $b$ for some real world problems. These are graphed in fig. 2. These plots show that for noise structures emerging from a discretized Brownian motion, like in the Asian option cases, the fit is very close. For noise structures extracted from market data, the fit is not that good. It is, however, the decline rate of the largest eigenvalues that influences the convergence rate most, and this shape is captured well by the functional relationship including $b$. Note furthermore that the eigenvectors should not have a large impact on the convergence rate as they have unit length.
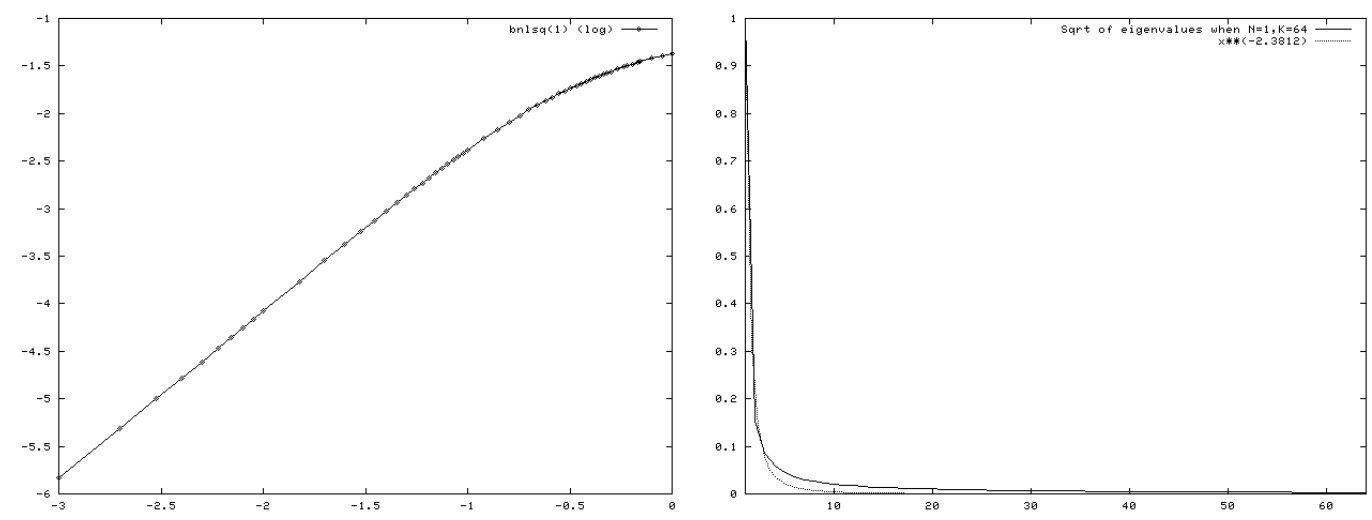

Figure1. In the left plot the parameter $b$ is given as a function of $\log _{10}(a)$. The dependence is close to linear. In the right plot a the square root of the eigenvalues (for $a=0.1$ ) and func. 9 (for the fitted b) is given for $D=K=64$ eigenvalues 

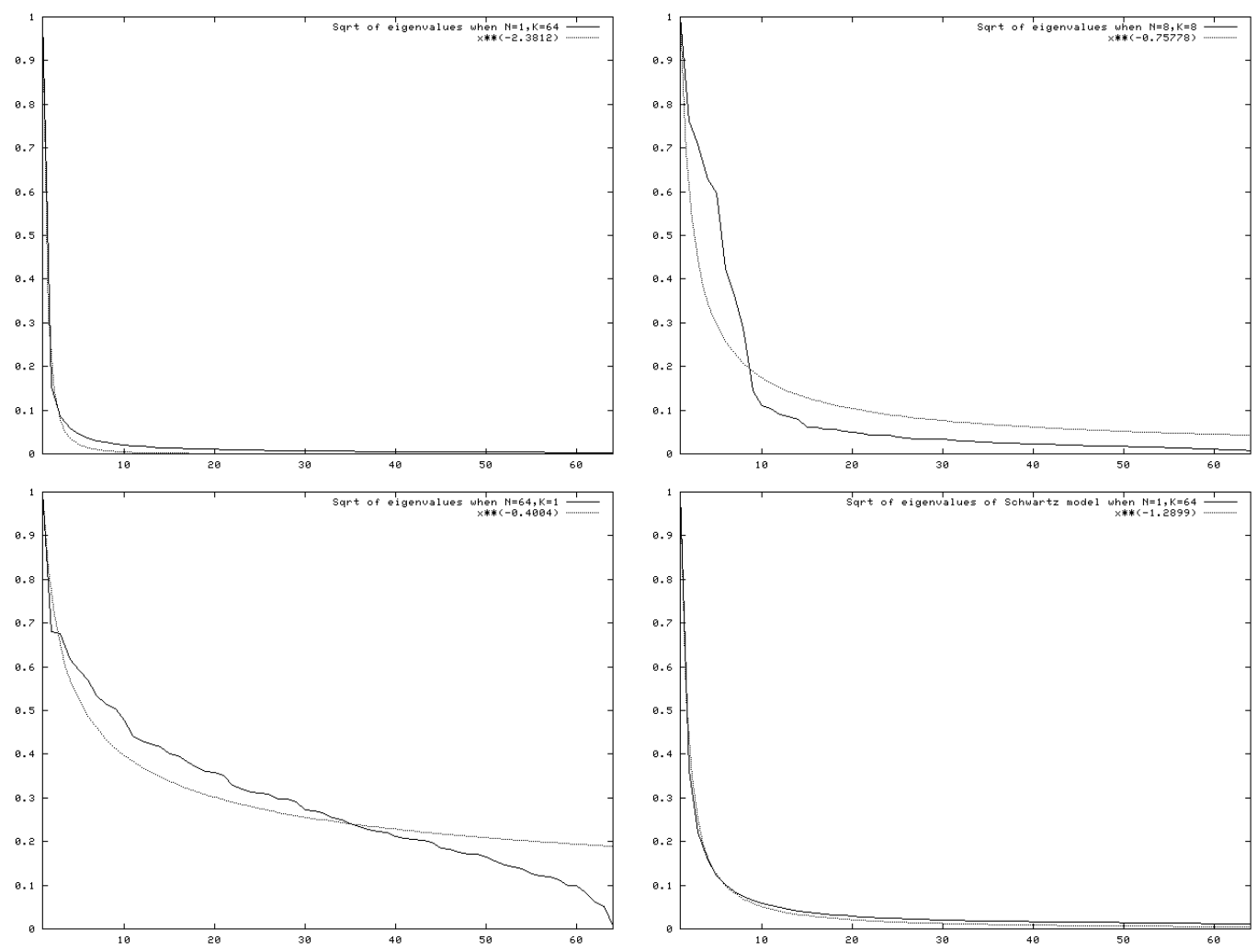

Figure2. The eigenvalues for the noise structure for a set of examples. The first three cases are for the Asian basket option case with $(N=1, K=64),(N=8, K=8)$ and $(N=64, K=1)$. The last example is from an asian option on a forward contract in the energy market, and $(N=1, K=64)$. We have used $a=0.1$ in eq. 8 for the propagation of time. As before $N$ is number of assets, while $K$ is number of time steps. We have included the appurtenant least-squares fit of func. 9 . On the $x$-axis is the dimension corresponding to the eigenvalues. On the $y$-axis is the square root of the normed eigenvalues

\section{$5 \quad$ Efficiency measures}

In order to compare the performance of the adaptive method with the conventional we need suitable measures. We focus on two such measures; one where the extra time consumption in the adaptive approach is accounted for, and one based purely on the difference in convergence speed in terms of variance. In detail I have measured the variance of 100 simulations of the integral by the adaptive and the non-adaptive for each combination of the parameters. The series of estimators for the adaptive method depend both on the distribution of points in each subdomain from the adaption process and on the ability of the method to sub-divide the domain well. In order for the variance $v_{a}$ of the 100 samples from the adaptive method and the variance $v_{n}$ of the conventional method to be comparable, the point estimates of the price are calculated by using different values $\delta$ for the subdivision accuracy in the adaptive method. This is to avoid using the exact same subdivision of the domain over and over, which would give the adaptive 
method an advantage when comparing with the non-adaptive. A specification of $\delta=10^{-3}$ as input to the simulation, therefore results in the adaptive method using $\delta \in\left[10^{-3}, 2 \cdot 10^{-3}\right]$ descending linearly as the simulations are performed to force different subdivisions for each estimated value. Furthermore it must be mentioned that the estimators are based on drawings from consecutive parts of the pseudo- and quasi-random number sequences for respectively the $\mathrm{MC}$ and QMC methods.

The first measure of efficiency, labeled DE (for definite efficiency) is constructed by the relation

$$
\mathrm{DE}=\log _{10}\left(v_{a} T_{a}\right)-\log _{10}\left(v_{n} T_{n}\right)
$$

where $T_{a}$ and $T_{n}$ are the computing times for resp. the adaptive and the conventional method. The DE-measure gives us a scale for the relative efficiency of the adaptive method to the conventional, where a negative number indicates preference for the adaptive method, while the same positive number gives an equivalent preference for the conventional. The scale is logarithmic, so a number of -1 would indicate that the adaptive was ten times better than the conventional, and so on. To see that the product of computing time and variance gives a good measure of efficiency for a method, consider the estimator $\hat{X}_{N}=\frac{1}{N} \sum_{i=1}^{N} X_{i}$ with $X_{i}$ i.i.d., $\mathrm{E}\left[X_{i}\right]=X$ and $\operatorname{Var}\left[X_{i}\right]=\sigma_{X}^{2}<\infty$. The central limit theorem asserts that as the number of replications $N$ increases we have

$$
\sqrt{N}\left(\hat{X}_{N}-X\right) \rightarrow \mathcal{N}\left(0, \sigma_{X}^{2}\right)
$$

Suppose that we use a fixed amount of computing time $\tau$ for each replication, then the number of replications we can calculate given a computational budget $\mathcal{T}$ is $\lfloor\mathcal{T} / \tau\rfloor$. This gives

$$
\sqrt{\mathcal{T}}\left(\hat{X}_{N}-X\right) \rightarrow \mathcal{N}\left(0, \sigma_{X}^{2} \tau\right)
$$

which suggests that, asymptotically, we should prefer an estimator having the lowest value of the product $\sigma_{X}^{2} \tau$. It should be mentioned that the above arguments are valid for truly stochastic variables only, and applicable for pseudo random variables. The same arguments may therefore not be used incautious to crate a measure for QMC methods (which are deterministic). QMC methods and their low discrepancy sequences are constructed with the purpose of improving the convergence beyond the $\sqrt{N}$ rate, An extensive literature on these subjects exist, see e.g. [15], [19], [18]. It can be shown that when a low discrepancy sequence is used, the convergence of an integral to its true value is proportional to the star discrepancy of the sequence multiplied by the variation of the integrand in the sense of Hardy and Krause. This is formalized through the Koksma-Hlawka theorem (see e.g. [11], [13], [14]). If we assume that the Koksma-Hlawka theorem can be interpreted similarly for the adaptive and the non-adaptive method, we 
can use the arguments above to justify the use of the DE-measure of efficiency also in the QMC setting. We can not, however, use the DE measure to compare calculation methods with differing underlying sequences. Therefore we will always construct the DE measure with common sequences for the underlying estimators.

The second measure of efficiency we have chosen to present, does not include the computing time. The background for presenting this measure, is that, although we have coded the algorithms as effectively as we could, there could still be ways to make them faster. The second measure therefore serves as comparison between the variances of a set of 100 replications of the adaptive method compared to the conventional. This measure is in the graphs denoted by $F$ (for fractional efficiency). It is calculated as

$$
F=\log _{10}\left(v_{a}\right)-\log _{10}\left(v_{n}\right) .
$$

A further aspect worth noting is that the efficiency-measures we have chosen are indifferent of the level of the integral itself: If we in order to normalize the integral, multiply by a factor of $d$, the variance will change by a factor of $d^{2}$. The estimators, however, are not affected since $\log _{10}\left(d^{2} v_{a}\right)-\log _{10}\left(d^{2} v_{n}\right)=$ $\log _{10}\left(v_{a}\right)-\log _{10}\left(v_{n}\right)$. Therefore the changes in the measures are due to the change of parameters, not other trivial effects. This is important, because the value of the integral will change as we change parameters of the option contract, and changes of parameters have to be done to reveal the characteristics of the integration methods.

\section{Numerical results of the parameterized problem}

In this section we will draw attention, and try to explain the main findings of the numerical simulations. We have simulated the price of the options by using different configurations in order to compare the performance of the adaptive algorithm with the conventional ${ }^{1}$ non-adaptive. For the market, we have used a risk free rate of 0.05 and a volatility of 0.5331 for the asset in the single asset Asian option (the volatility of $\mathrm{Kv} æ \mathrm{rner}^{2}$ in 2000). The varied parameters of the model are the number of time discretization steps $(K):\{2,64\}$, moneyness $(m)$ : $\{-25,0,25,50\}$, sequence generator: (method) $\{\mathrm{H}, \mathrm{M}\}$ (Halton Leaped, Mersenne Twister), Control variate technique: (CV) $\{\mathrm{Y}, \mathrm{N}\}$ (yes, no), importance sampling: (IS) $\{\mathrm{Y}, \mathrm{N}\}$ (yes,no), the parameter $a$ in eq. $8:(a):[0.001,1]$ - leading to a decline rate for the square root of the eigenvalues in the range $[-5.88,-1.37]$ (see sec. 3 on the relation between $a$ and $b$ and how we find an extended range for $b$ ). In the figures these different parameters are varied and plotted against each other. We

\footnotetext{
${ }^{1}$ Note that the label conventional is here used on a highly sophisticated method using principal component analysis (SVD) to find the most important dimensions of the noise sources combined, and using a low discrepancy sequence as uniform number generator

${ }^{2}$ Kværner is one of the largest companies in the main index of Oslo stock exchange, and had a rather high volatility in 2000
} 
have also varied the variance estimator limit $\delta$, which controls the subdivision coarseness, $(\delta):\left\{10^{-2}, 10^{-3}, 10^{-4}\right\}$. Note that when the DE - efficiency measure is negative, the adaptive method has the best performance. In the relevant plots, we have included the horizontal line at 0 to ease the reading of the graphs.
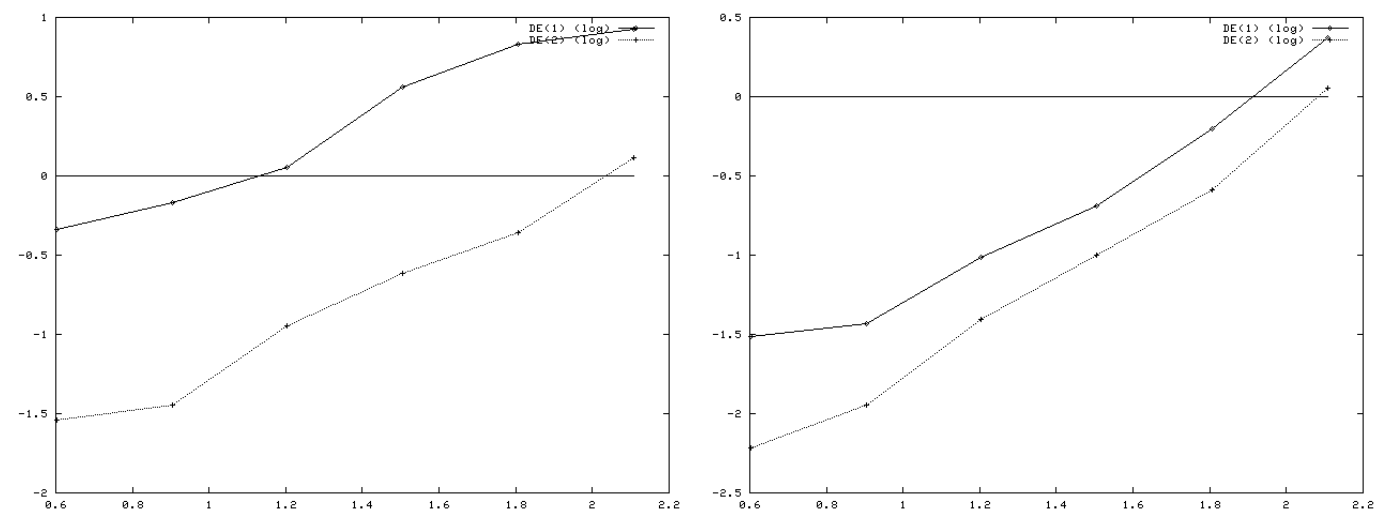

Figure3. Plot of DE-efficiency vs. $\log _{10}(D)$ (dimension) for $b=-1.55(1)$ and $b=-2.38$ (2) (equivalent to $a=0.5$ and $a=0.1$ ). In the left figure is the Halton Leaped sequence used, while the Mersenne Twister is used in the right
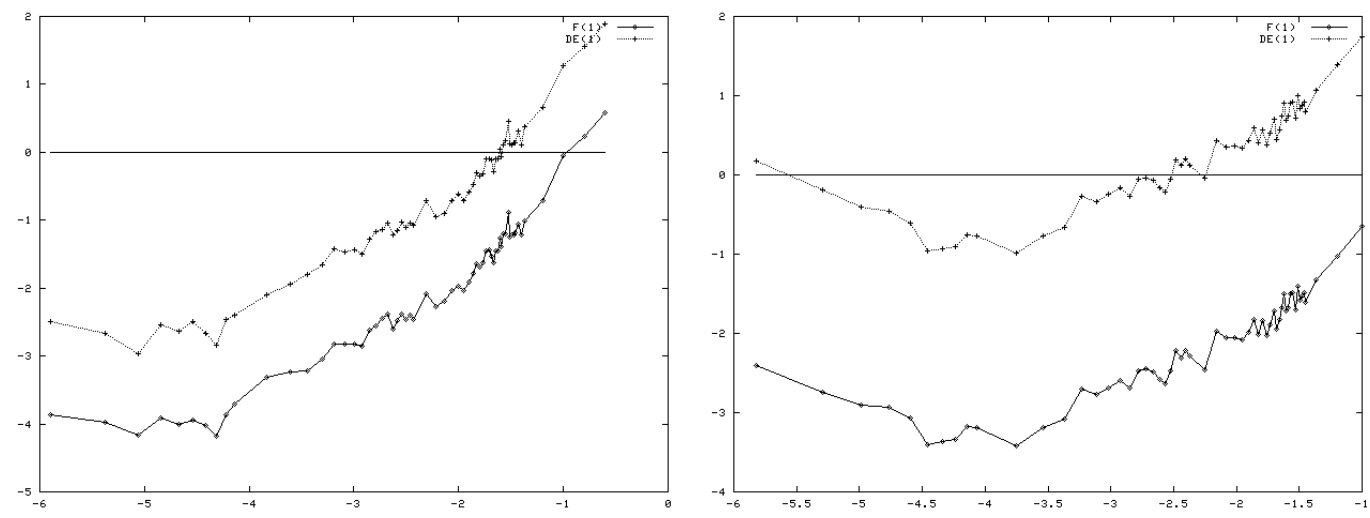

Figure4. Plot of both efficiency measures $\left(F\right.$ and DE) vs. $\log _{10}(b)$ (eigenvalue decline rate) for a typical case. In the left figure $D=16$ and in the right $D=128$

First of all, the dimension of the problem is important for the relative performance, and the ability of the adaptive method to outperform is better for lower dimensions (fig. 3). Furthermore the adaptive method is relatively better for problems where the eigenvalues decline rapid, and for out-of-the-money options (figs. 4, 5). These findings are connected, and the explanation for them are twofold: first it is evident that the adaptive method is able to exploit that the integrand has value zero in large areas of the domain. This explains why out-of- 

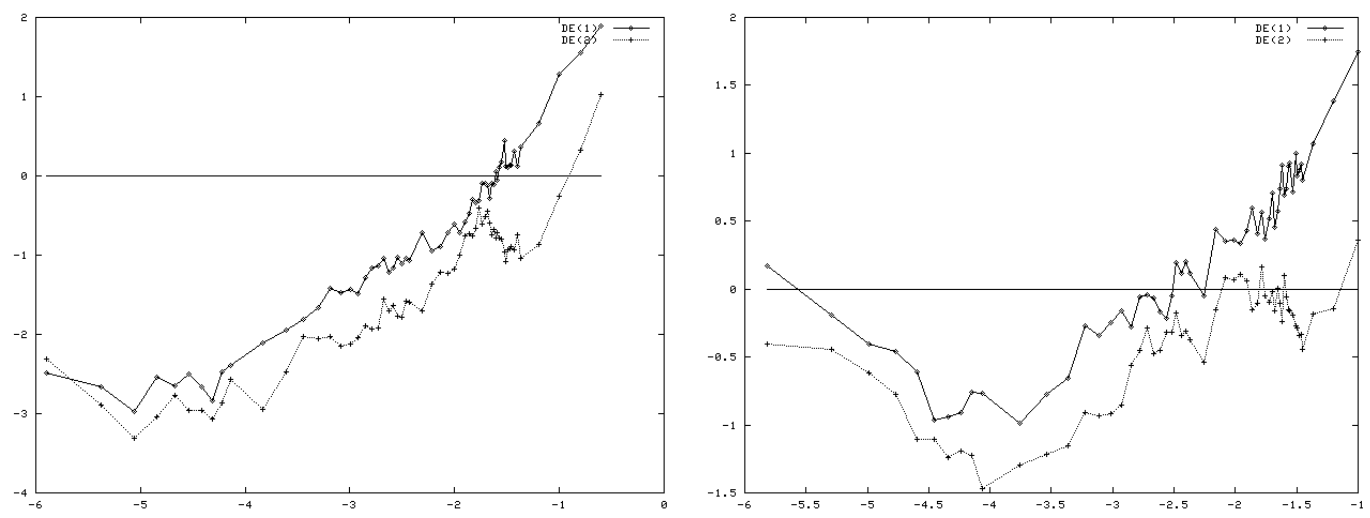

Figure5. Plot of DE-efficiency vs. $b$ for different moneyness - (1) corresponds to moneyness $=0$, while (2) corresponds to moneyness $=50$. In the left figure $D=16$ and in the right $D=128$

the-money option are easier for the adaptive method than in-the-money options (fig. 8). A rapid declining rate of the eigenvalues tells us that the contribution to the variance of the integral is limited to a small set of dimensions of the integrand, and the adaptive method is able to exploit this. Further arguments for the connection between the effective dimension of the problem and the behavior of the eigenvalues can be found in [5] where we have discussed and measured the relation between the eigenvalues of the noise of the problem and the number of contributing dimensions, and concluded with a close relationship.

Next we turn to the question; why does the ability to outperform decrease with increasing dimension (fig. 3)? The answer to this lies in the curse of dimensionality: The hyper-surface of the cube increases as the dimension increases while the volume is constant equal to 1 . This results in the fraction of volume where the integrand has value zero decreases as the dimension of the problem increases if the fraction of important dimensions does not decrease. This is because the fraction of the volume lying close to the borders of the domain is increasing as the number of dimensions increases. To concretize; the volume fraction of the $[0.01,0.99]^{2}$ cube is about 0.96 relative to the $[0,1]^{2}$ cube, while the volume fraction of the $[0.01,0.99]^{64}$ cube relative to the $[0,1]^{64}$ cube is about 0.27 . The same argument in dimension 1024, which is a realistic case with 32 assets and 32 time discretization points, gives a fraction of about $10^{-9}$. The nested exponential functions in the integrand in option pricing problems results in dominance for a variable as it goes to the border, so by the argument above we have a situation where the adaptive method looses an important advantage as the number of contributing dimensions increase.

The local control variates technique enhance the performance only for problems having the most rapid decline of the eigenvalues, (fig. 6). This is probably due to the failure of generating a polynomial function behaving like the original function for the option pricing integrand, even for very local domains, as 

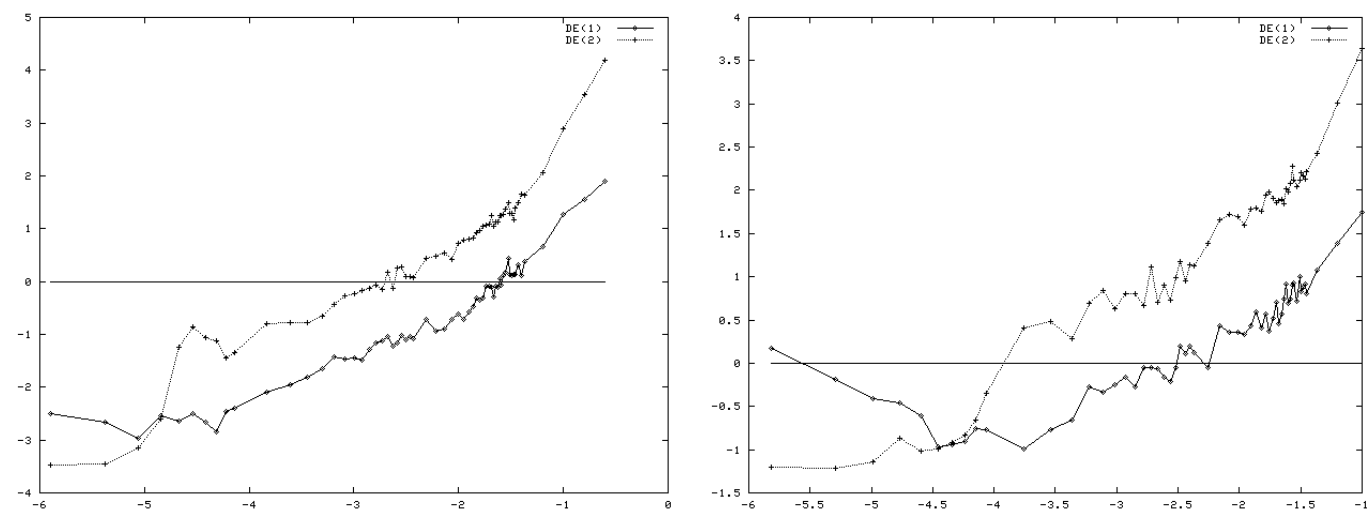

Figure6. Plot of DE-efficiency vs. $b$ with the control variate technique (label (2)) and without (label (1)). In the left figure $D=16$ and in the right $D=128$
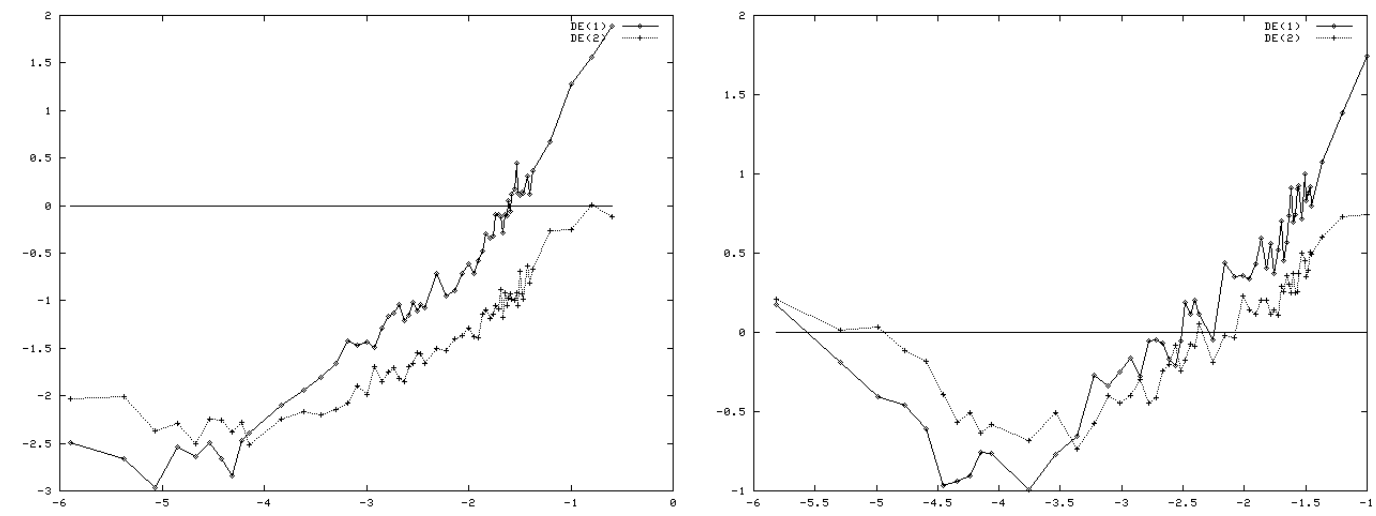

Figure7. Plot of DE-efficiency vs. $b$ by using different uniform number generators - (1) corresponds to the Halton Leaped sequence, while (2) corresponds to the Mersenne Twister. In the left figure $D=16$ and in the right $D=128$

the dimension is increased. Note, however, that for less complicated integration problems we have experienced that the control variate technique gives a huge performance gain. In sec. 8 we give some numerical results showing this.

There can be various reasons for choosing a plain MC method when pricing options, and the adaptive method can be used in this setting as well. The findings of our numerical tests are that the relative performance of the adaptive method is better in the MC setting than in the QMC setting (fig.7). Note, however, that the QMC estimators gives the most accurate results for both the adaptive and the conventional method, but because the non-adaptive MC method has considerably lower performance than the corresponding QMC method the relative performance is better in the MC setting. See e.g. [2] or [4] for illustrations on the performance differences between $\mathrm{MC}$ and QMC.

It is interesting to note that as the variance estimator limit $C$, which controls the subdivision coarseness, is changed, the measures of relative efficiency 

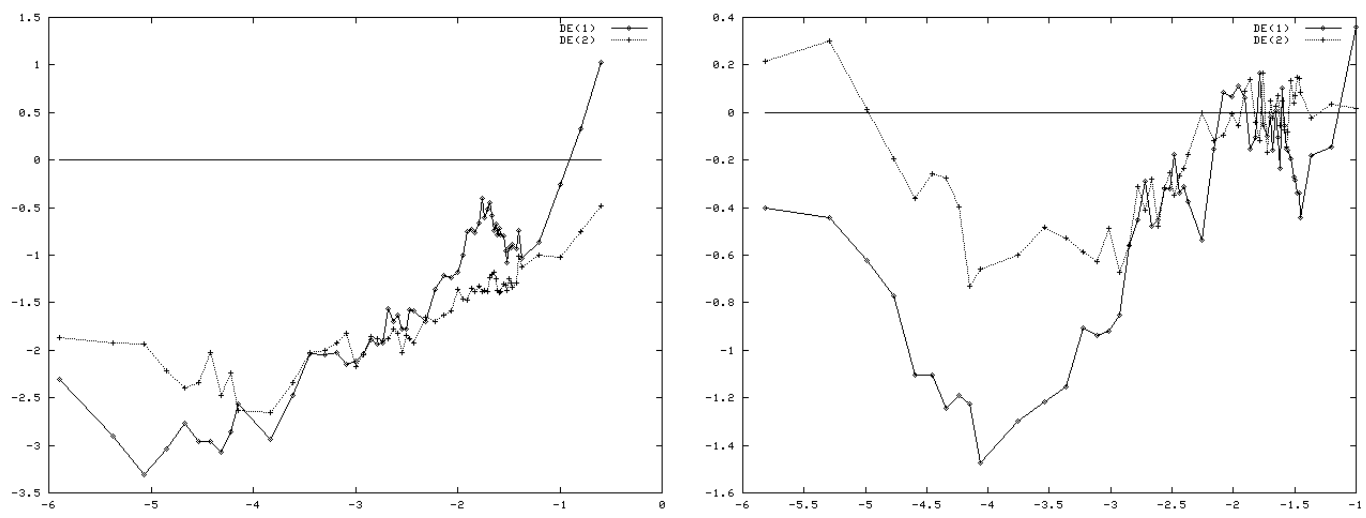

Figure8. The same comparison as in fig. 7, but now we do the plots for moneyness $=50$ rather than moneyness $=0$. In the left figure $D=16$ and in the right $D=128$
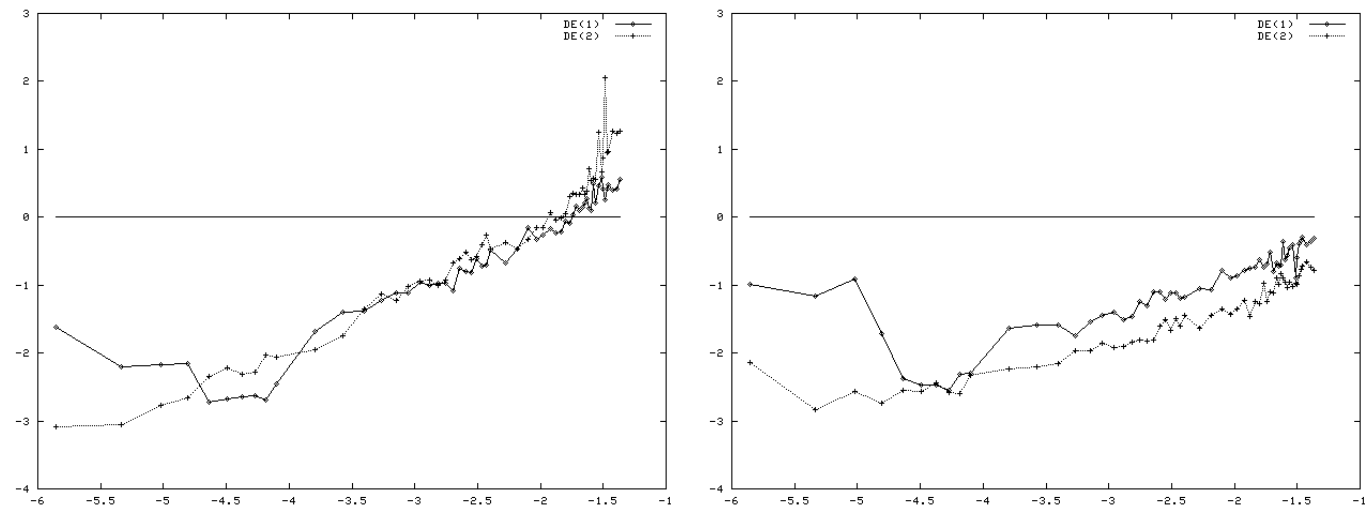

Figure9. Plot of DE-efficiency vs. $b$ for $\delta=10^{-3}$ in label (1) and $\delta=10^{-4}$ in label (2). The dimension of the problem is $D=32$. In the left figure the Halton Leaped sequence is used, while in the right the Mersenne Twister

is changed in a different way when the Halton sequence is used and when the Mersenne Twister sequence is used. In the QMC setting the relative efficiency only changes for the set of problems with a very high decline rate $(b<-4.5)$, while it gets better for all problems in the MC setting. The second observation reveals that the adaptive method gains more from adding computational work than the non-adaptive when a pseudo random sequence is used, while the first observation means that this is only the case for the QMC setting for $b<-4.5$. For $b>-4.5$, the relative efficiency is the same. An illustration of these results is given in fig. 9 .

To investigate the effect of applying importance sampling, we have to modify the efficiency measures. The background for this is that by applying importance sampling we modify the integrand in such a way that the adaptive method will act differently on the problem. This results in a different subdivision and a different number of simulation points than for the simulation on the non-altered problem. 

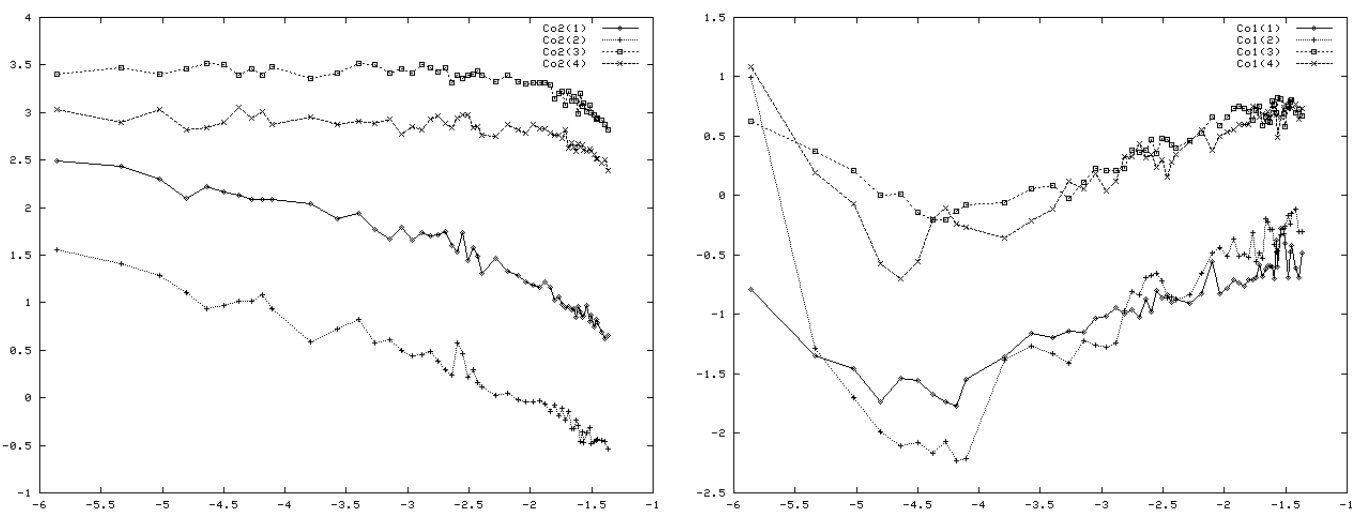

Figure10. Plot of $E_{n}$-efficiency vs. $b$ for respectively no importance sampling and QMC (1), importance sampling and QMC (2), no importance sampling and MC (3) and importance sampling and MC(4). In the right plot is the corresponding graphs for the measure $E_{a}$ (for the adaptive method). The dimension is $D=32$

The DE-measure is a compound measure and can still be used in the importance sampling (IS) setting to find the effect of applying the adaptive method to an IS modified problem, but we must use less compound measures if we want an answer to whether we have benefitted by applying importance sampling in the first place. This situation is different from what was the case when applying the control variate technique, which only affected the treatment of the integrand in the adaptive setting. We therefore introduce the non-relative measures $E_{a}=\log _{10}\left(L v_{a}\right)$ and $E_{n}=\log _{10}\left(L v_{n}\right)$ for respectively the adaptive and non-adaptive method. They can be used to compare the effects of changing parameters and variance reduction techniques, but not to compare the effect of changing method. The main finding is that the performance of the adaptive method is virtually unchanged by applying importance sampling. The case for the non-adaptive method, however, is different. Here the insertion of the IS technique has a positive effect for $D \leq 32$. Furthermore, the variance of the estimator did not increase by applying importance sampling for any of our simulations. The time consumption, however, increased as a consequence of the fixed-point iteration. It is interesting to note that the adaptive method had the ability to neutralize the effect of importance sampling. Note that the importance sampling technique, which is applied to the problem globally, is only practical for integration problems of probability density functions and can not be applied to the functions in sec. 8 .

\section{Numerical results of actual pricing problems}

In sec. 4 we found that many option pricing problems have an eigenvalue structure that can be imitated by the simple function $l(i)=i^{b}$, for some $b$. In this section we are going to simulate the value of a European basket option with $N=8$ underlying assets to check if the resulting values for $(b, \mathrm{DE})$ are in accordance with 
the ones we have predicted by simulating on the corresponding parameterized Asian option problem with $K=8$ time discretization points. The results are given in fig. 11, and shows a good match.
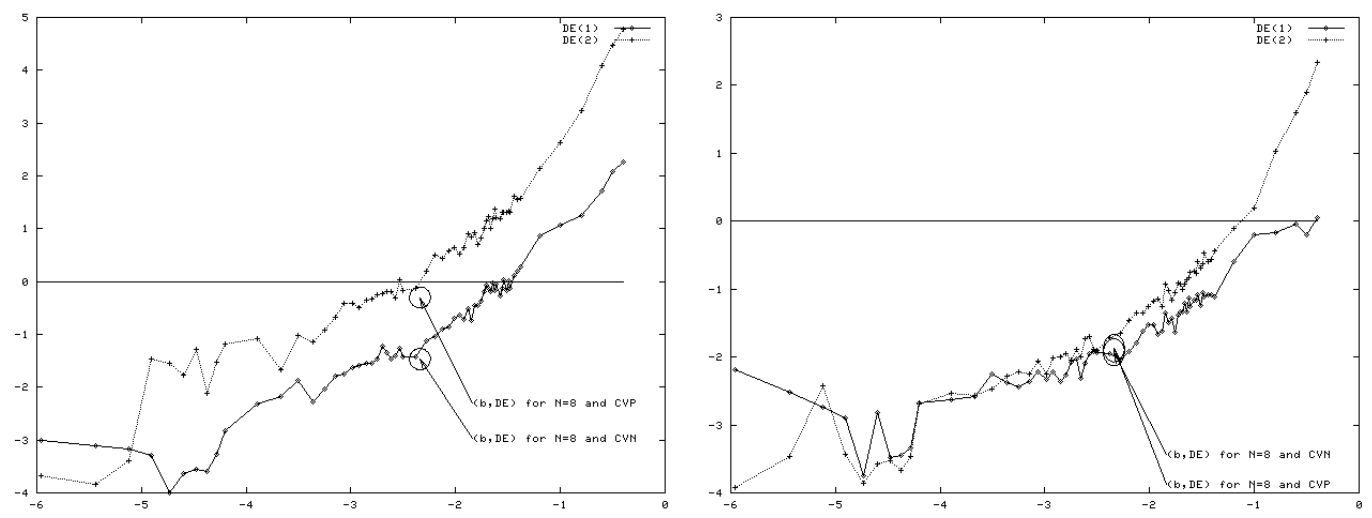

Figure11. Plot of DE-efficiency vs. $b$ with the control variate technique in label (2) and without in (1). Included in the figures are the coordinates of a basket option problem simulated with the same methods. The dimensions of the problems are $D=8$. In the left figure the Halton Leaped sequence is used, while in the right the Mersenne Twister

\section{Additional test functions}

We have included two additional test functions, with less resemblance with the option pricing problem. This is to show that the control variate technique can be a valuable method for various integration problems, even if it did not perform very well in general for the option pricing problems. Also for these functions a parameter $b$ is added in order to control fluctuations of the integrand for increasing dimensions. Note, however, that there are no close relationship between the interpretation of $b$ for these functions and for the problem in sec. 3, except that it is used to parameterize the behavior of the integrand in different dimensions. The functions are given by

$$
\begin{aligned}
& f_{1}(x)=\sum_{i=1}^{D} i^{b}\left(1+\mathrm{e}^{\left(\pi x_{i}\right)}\right), x \in[0,1] \\
& f_{2}(x)=D \exp \left(\frac{1}{D} \sum_{i=1}^{D} i^{b} 6 x_{i}\right), x \in[0,1]
\end{aligned}
$$

where $b \in[-4,-1]$. Simulations on these functions shows that the control variate technique enhance the adaptive method considerably (figs. 12 and 13), and that the gain from applying $\mathrm{CV}$ is increasing as $b$ is decreasing. 

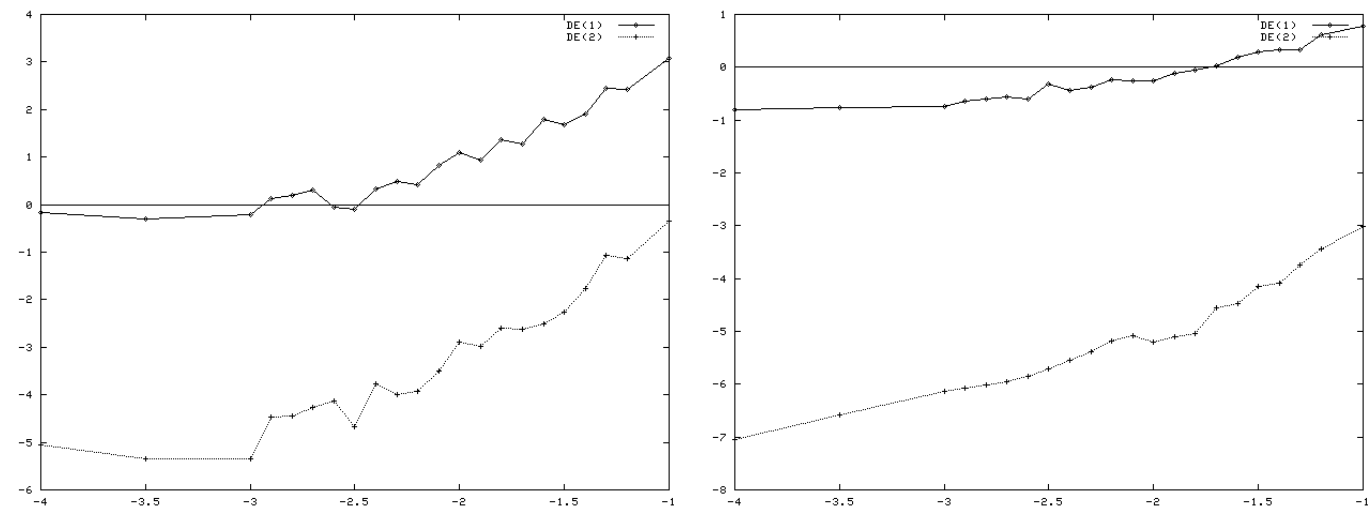

Figure12. Plot of DE-efficiency vs. $b$ with the control variate technique in label (2) and without in (1) for the function $f_{2}$. The dimensions of the problem is $D=32$. In the left figure the Halton Leaped sequence is used, while in the right the Mersenne Twister
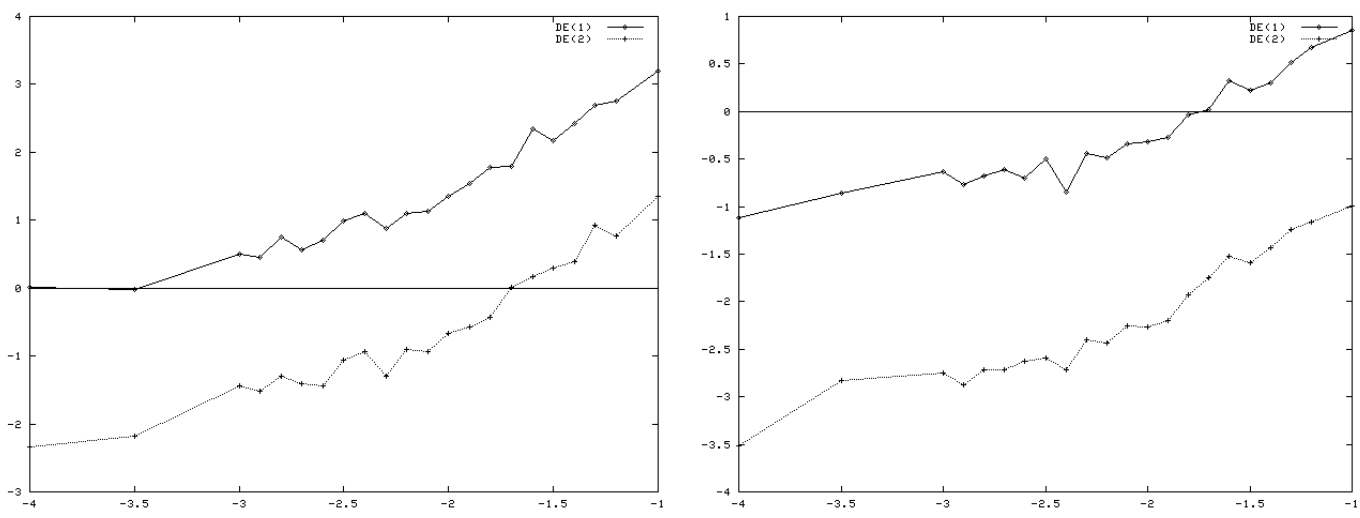

Figure13. Plot of DE-efficiency vs. $b$ with the control variate technique in label (2) and without in (1) function for $f_{1}$. The dimensions of the problem is $D=32$. In the left figure the Halton Leaped sequence is used, while in the right the Mersenne Twister

\section{Conclusion and discussion}

It is rather obvious that an adaptive QMC integration method for some problems will outperform a non-adaptive. And the integrands for which this will be the case have to be sufficiently nice in parts of the integration domain to allow the more sophisticated method to come out ahead. We wanted to find the set of option pricing problems where this was the case, and criteria for pinpointing these problems in advance. The criteria were formulated through a parameterized function fitted to the sorted eigenvalues in descending order. Furthermore we established an indirect method for altering the parameter. This gave us an apparatus to investigate the performance of the adaptive method compared to the non-adaptive for different parameters of the problem, and for various sophistication levels of the methods. The major part of this article focuses on presenting and elaborating 
on these results. In the companion article [3] the method itself and the sophistication levels are developed. We do not include any of the detailed findings in this section, as most of the article is dedicated to this, but limit ourself to stating that we have found that the adaptive method can give considerable performance gains for many problems. In general it will be the method of choice for low dimensional problems, and for higher dimensional problems it can give considerable performance gains for problems with certain characteristics. It should, however, not be used incautious for high dimensional problems, since the performance also can deteriorate for problems not having these characteristics.

It should be mentioned that a vast set of simulations has been performed, summing up to about 6000 hours of computing time on a cluster of PC's with average CPU speed of about $1 \mathrm{Ghz}$. Additional and deepening results can therefore be obtained by contacting the author.

\section{Acknowledgements}

The author is grateful for financial support from Storebrand Investments. I also want to thank F. E. Benth for valuable talks and for applying amendments to the manuscript.

\section{References}

1. J. Berntsen, T. O. Espelid, and A. Genz. An adaptive algorithm for the approximate calculation of multiple integrals. ACM Transactions on Mathematical Software, 1991.

2. L. O. Dahl. Valuation of European call options on multiple underlying assets by using a quasiMonte Carlo method. a case with baskets from Oslo stock exchange. In Proceedings AFIR 2000, volume 10, pages 239-248, June 2000. .

3. L. O. Dahl. An adaptive method for evaluating multidimensional contingent claims. Part I. Submitted to International Journal of Applied and Theoretical Finance. Currently in preprint at http://www.math.uio.no/eprint/pure_math/2002/pure_2002.html. , December 2001.

4. L. O. Dahl and F. E. Benth. Valuation of Asian basket options with quasi-Monte Carlo techniques and singular value decomposition. Submitted to Journal of Computational Finance. Currently in preprint at http://www.math.uio.no/eprint/pure_math/2001/pure_2001.html., February 2001.

5. L. O. Dahl and F. E. Benth. Fast evaluation of the Asian basket option by singular value decomposition. In K.-T. Fang, F.J. Hickernell, and H. Niederreiter, editors, Monte Carlo and Quasi-Monte Carlo Methods 2000. Springer-Verlag, Berlin, 2002. .

6. P. Glasserman, P. Heidelberger, and P. Shahabuddin. Gaussian importance sampling and stratification: Computational issues. In D. J. Medeiros, E. F. Watson, J. S. Carson, and M. S. Manivannan, editors, Proceedings of the 1998 Winter Simulation Conference, volume 1, pages 685-693. IEEE Computer Society Press, September 1998.

7. P. Glasserman, P. Heidelberger, and P. Shahabuddin. Asymptotically optimal importance sampling and stratification for pricing path-dependent options. Mathematical Finance, 9(2):117-152, 1999.

8. P. Glasserman, P. Heidelberger, and P. Shahabuddin. Importance sampling in the Heath-JarrowMorton framework. Journal of Derivatives, 7(1):32-50, 1999.

9. P. Glasserman, P. Heidelberger, and P. Shahabuddin. Importance sampling and stratification for value-at-risk. In Computational Finance 1999 (Proceedings of the Sixth International Conference on Computational Finance), pages 7-24. MIT Press, 2000.

10. I. Karatzas and S. E. Shreve. Methods of Mathematical Finance. Springer Verlag, 1998.

11. L. Kuipers and H. Niederreiter. Uniform Distribution of Sequences. John Wiley and Son, 1974.

12. B. Moro. The full monte. Risk, (8):57-58, February 1995. 
13. H. Niederreiter. Quasi-Monte Carlo methods and pseudo-random numbers. Bulletin of American Mathematical Society, 84:957 - 1041, 1978.

14. H. Niederreiter. Point sets and sequences with small discrepancy. Monatsh. Math., 104:273 - 337, 1987.

15. H. Niederreiter and C. Xing. The algebraic-geometry approach to low-discrepancy sequneces. In Monte Carlo and Quasi-Monte Carlo Methods 1996, number 127 in Lecture Notes in Statistics, pages 139-160. Springer Verlag, 1996.

16. B. Øksendal. Stochastic Differential Equations, An Introduction with Applications. Springer Verlag, 1998.

17. A. Owen and Y. Zhou. Safe and effective importance sampling. Journals of the American Statistical Association, 95(449):135-143, 2000.

18. A. B. Owen. Latin supercube sampling for very high dimensional simulations. ACM Transactions on Modeling and Computer Simulation, 8(1), 1998.

19. A. B. Owen. Monte Carlo extension of quasi-Monte Carlo. Technical report, Department of Statistics, Stanford University, 1999.

20. W. H. Press and G. R. Farrar. Recursive stratified sampling for multidimensional Monte Carlo integration. Computers in Physics, 1990.

21. L.C.G Rogers and Z. Shi. The value of an Asian option. Journal of Applied Probability, 32:10771088, 1995.

22. F. J. Vázquez-Abad and D. Dufresne. Accelerated simulation for pricing Asian options. In D. J. Medeiros, E. F. Watson, J. S. Carson, and M. S. Manivannan, editors, Proceedings of the 1998 Winter Simulation Conference, 1998. 